\title{
THE ROLE OF THE FOOT IN THE MECHANISM OF SHOCK ABSORPTION
}

\section{Tamás Kobezda}

Department of Anatomy, Medical and Health Science Center, University of Debrecen tamasemailcime@gmx.net

\begin{abstract}
The present work introduces an anatomical specimen of the muscles of the foot with the intention to promote the understanding of its structure in anatomical courses. The specimen is preserved with the plastination technique of Günther von Hagens. Special attention has been paid to the potential function of individual pedal muscles in the support of the plantar arches as deduced from the fascicular geometry and attachment of the muscles. Attention has been called to the importance of the care of the foot in order to maintain its structural and functional integrity.
\end{abstract}

Keywords: arches of the foot, intrinsic foot muscles, foot care

\section{Introduction}

In addition to its several advantages, the bipedal locomotion has a serious handicap which concerns primarily the head. When the body tilts from the upright position, the head moves along an arc with the centre on the resting foot, and the height of the body is the radius of the arc. The acceleration of the movement along the arc increases proportionately with the length of the radius, thus the weight of mass of the head increases accordingly. The brain floats in a fluid in the cranial cavity, but the thin layer of the surrounding cerebrospinal fluid does not give a secure protection against sudden changes in acceleration (c.f. car accidents). If our body would be a solid frame and we jumped down from a height of half a metre, the negative acceleration at landing would exceed the force of gravity, and our head would hit the cervical vertebral column with a force that would be just sufficient to break through the basis of the occipital bone. A similar consideration may be made that we could not make a single step without the threat of an imminent commotion. It is obvious that the bipedal locomotion requires a series of shock absorption structures.

Between the skull and the ground, the first shock absorption structure is represented by the vertebral column. Of the curvatures of the column themselves, one may expect a certain amount of springing, but an effective shock absorption is secured by the outer fibrous shell (annulus) and the inner pulpy, highly elastic substance (nucleus) of the intervertebral discs. The next shock absorber is at the bilateral sacroiliac articulations. The vertebral column conveys the weight of the upper part of the body to the basis of the sacral bone, and pushes it into the cavity of the pelvis. The sacral bone floats, as a two-armed lever, in the dorsal arch of the pelvic girdle, and the posterior arm of the lever is balanced by very strong ligaments in the sacroiliac joints. The principal task of the lower limbs is to secure a solid and springy support for the body and to allow large freedom of movement at the same time. The interplay of the flexor-extensor muscle groups of the knee joint renders fine buoyant 
steps possible in the act of walking. The entire load of the body project onto the foot; and to answer the task of smooth movements the foot requires a strong and solid structure with large freedom of movement, and an efficient shock absorption mechanism. Many important details about the anatomy of the foot are conscientiously described in a number of textbooks, still I have been wondering if in addition to the standard anatomical courses I would be able to find any structural attribute which makes the synchronous performance of the three opposing actions possible: solidity, mobility and elasticity. I have been also interested whether it would be possible to propose any new technique for the care of this complex structure. Structures of shock absorbers require caretaking, and unlike other structures, we can, moreover we have to take care for the healthy structure of our foot.

\section{Material and technique}

The dissection was performed on the foot attached to the distal one third of the leg and fixed in a solution of $4 \%$ formalin. Following preparation and cleaning the superficial and deep structures, preliminary steps were made for the preservation of the specimen. The technique of plastination described by von Hagens ${ }^{6}$ was chosen, because this procedure keeps the form and position of structures with a minimum shrinkage in a dry anatomical specimen. The different structures can be coloured with common enamel paints. The procedure is as follows. Dehydration is made in three changes of aceton at minus 25 degrees for 3 to 5 weeks, depending on the size of the specimen. The low temperature helps stabilize the shape of the structure. This is followed with acetone treatment at room temperature for 2-3 days to remove fat. The next step is impregnation, that is, the replacement of acetone with a special polymer (BIODUR) compounded by the author. To achieve a complete impregnation it is advisable to perform it in vacuum $(2-15 \mathrm{Hgmm})$. The final step is hardening which, depending on the type of the polymer, can be done with light, heat, or with the appropriate gas. Before hardening the specimen can be moulded to the desired form.

\section{The description of the anatomical preparation}

After removal of the skin from the distal part of the leg, subcutaneous structures: the great and the small saphenous veins, the saphenous and the sural nerves come to sight on their usual sites (Figure 3). Tracking the veins leads us to

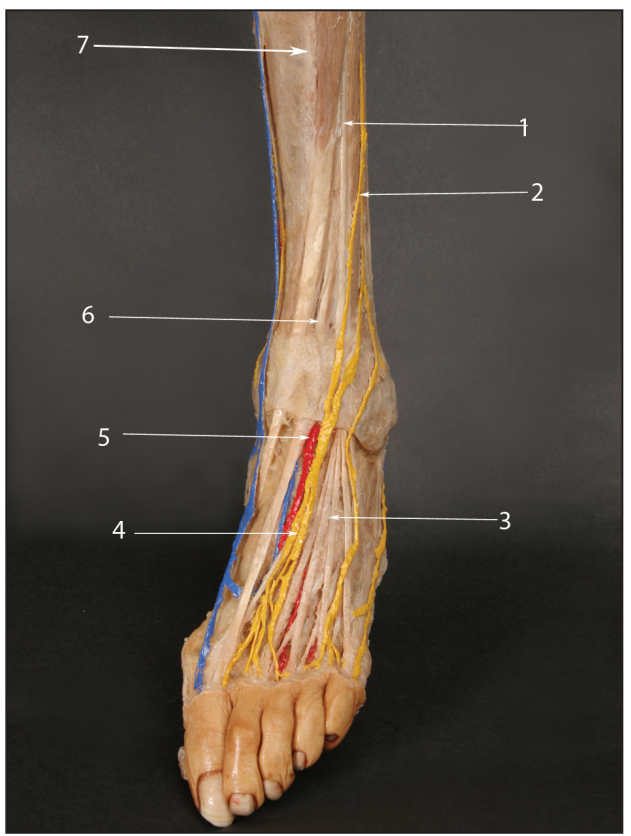

Figure 1. The distal part of the leg and the dorsum pedis

$1: \mathrm{m}$. extensor digitorum longus; -2 : $n$. peroneus superficialis; -3 : tendons of the extensor digitorm longus muscle; -4 : n. cutaneus dorsalis pedis; 5: a. \& v. dorsalis pedis; $-6: \mathrm{m}$. extensor hallucis longus; -7 : $\mathrm{m}$. tibialis anterior 


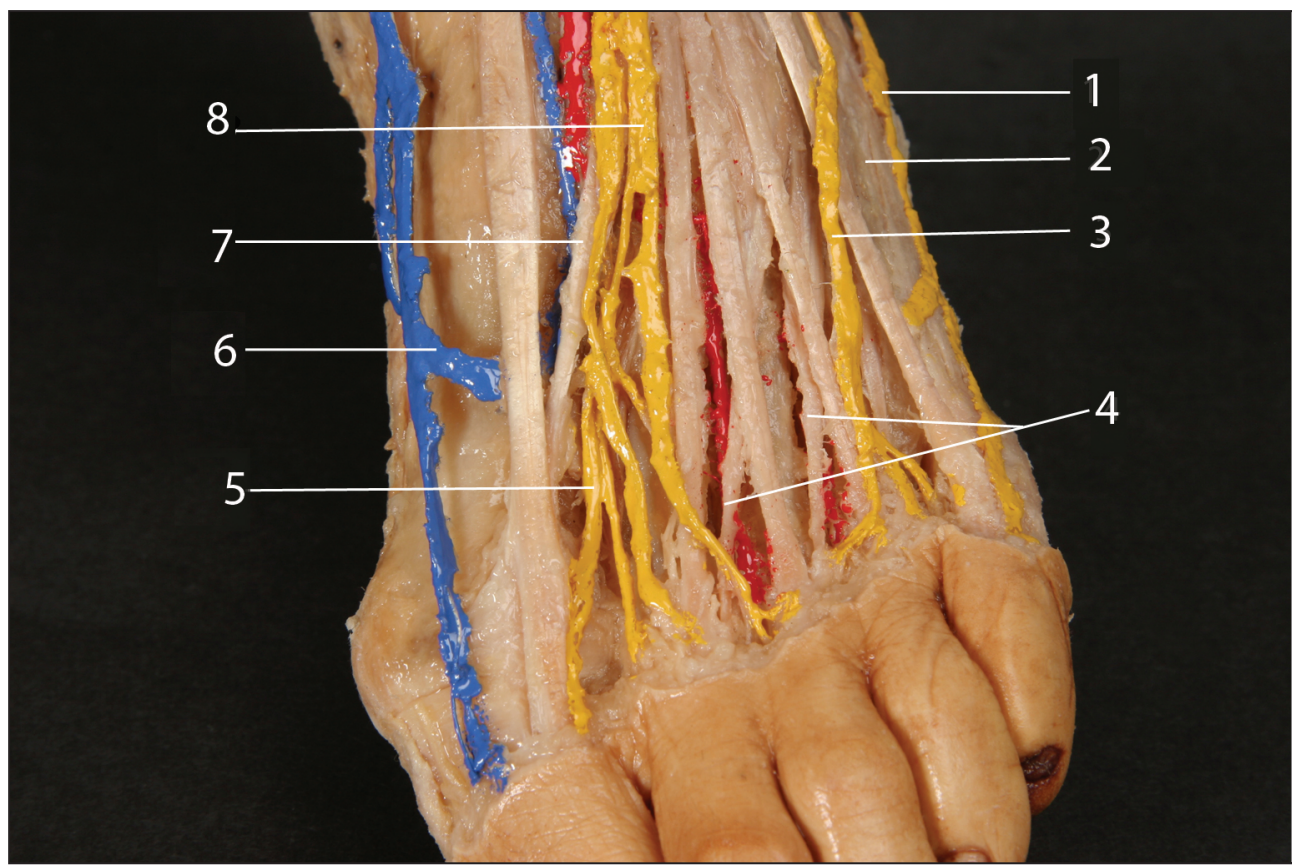

Figure 2. The distal part of the dorsum pedis

1: $n$. cutaneus dorsalis pedis lateralis; $-2: \mathrm{m}$. abductor digiti minimi; $-3: \mathrm{n}$. cutaneus dorsalis pedis intermedius; -4 : $\mathrm{m}$. extensor digitorum brevis; -5 : terminal branch of the deep peroneal nerve; 6: venous arch of the dorsum pedis; $-7: \mathrm{m}$. extensor hallucis brevis; -8 : $\mathrm{n}$. cutaneus dorsalis pedis medialis

the venous arc, and in the continuation of the sural nerve, the intermedial and lateral cutaneous nerves appear on the dorsum of the foot (Figure 2).

On the dorsum of the foot, the tendons of the anterior tibialis muscle and the long extensor of the toes, and under them the belly of the short extensor muscle of the toes can be identified (Figure 2). The bellies of the muscles are in the leg, where they are swaddled in four fascial compartments. In the anterior compartment, the anterior tibial, the long extensor of the hallux and the long extensor of the toes can be found. The lateral compartment is shared by the two peroneal muscles, the long and the short. The superficial posterior compartment contains the big triceps surae muscle, and the deep posterior compartment houses the poste- rior tibial muscle, the long flexor muscles of the toes and of the hallux. The tendons of these muscles bend to the sole behind the medial ankle (Figure 3). The tendon to the hallux

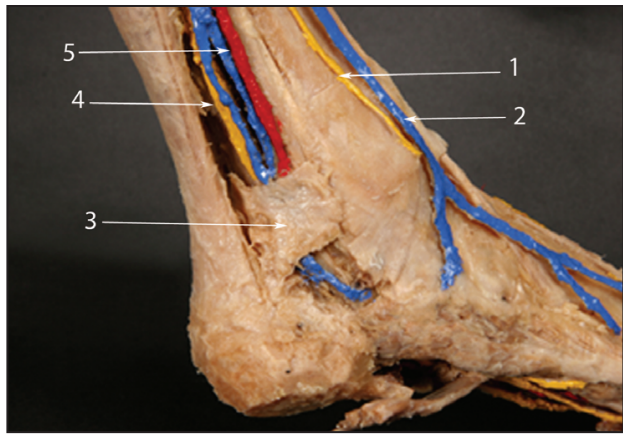

Figure 3. Regio malleolaris medialis

1: n. saphenus; -2 : v. saphena; -3 : retinaculum musculorum flexorum; -4 : n. tibialis; 5: a. \& v. tibialis posterior. 
runs underneath a medial shelf of the calcaneus (sustentaculum tali) and plays an important role in the support of the talus.

The posterior tibial artery and its nerve companion also enter the sole behind the medial ankle. The anterior tibial artery emerges from the anterior compartment, runs on the dorsum of the foot and ends in the dorsal foot arc. Its nerve companion is the deep peroneal nerve which terminates with two branches between the facing surfaces of the hallux and the first toe (Figure 2).

The removal of the skin from the sole together with the thick panniculus adiposus exposes the strong aponeurosis plantaris. It tightly covers the muscles and with two septa confines them into three compartments. The aponeurosis must be entirely removed to expose the muscles which are arranged in four layers. In the superficial layer, the strong short flexor of the toes is immediately prominent, and it is flanked by the abductor hallucis medially, and by the abductor of the fifth toe laterally (Figure 4). The flexor muscle of the toes is separated from the calcaneus and its distal part is folded forward to the tip of the toes. The prominent mass of the hallux abductor suggests strength and it plays a major role at the beginning of the swing phase in the act of walking.

In the next layer the short flexor of the hallux and the flexor of the fifth toe are partially covered by the above lying short flexor of the toes (Figure 4; 11, 6). Between these short flexors the tendons of the long flexor of the toes stretch in intact foot; they are cut in the present specimen and folded backward toward the calcaneus. The qudratus plantae can be seen attached to the tendon of the long flexor, and on the distal remaining segments of the tendons the lumbrical muscles can be observed (Figure 4; 3, 9). Muscles constituting the

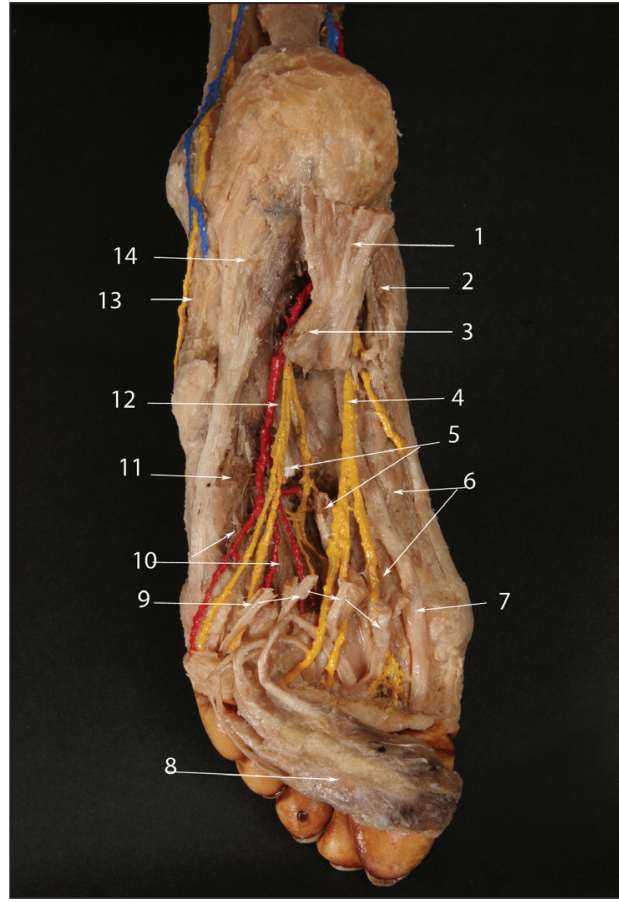

Figure 4. Plantar structures of the foot.

1. $\mathrm{m}$. flexor digitorum longus (bent back); -

2: $\mathrm{m}$. abductor hallucis; $-3: \mathrm{m}$. quadratus plantae; - 4: medial branch of the plantar nerve; 5: oblique head of the adductor hallucis muscle (the middle part removed); $-6: \mathrm{m}$. flexor hallucis brevis; - 7: $\mathrm{m}$. flexor hallucis longus; -

8: $\mathrm{m}$. flexor digitorum brevis (bent forward); -

9: $\mathrm{mm}$. lumbricales; - 10: $\mathrm{mm}$. interossei plantares; - 11: m. flexor digiti minimi; 12: a.\& n. plantaris lateralis; -13 : m. opponens digiti minimi; - 14: $\mathrm{m}$. abductor digiti minimi

medial and lateral eminences are only partially explored, the oblique head of the adductor hallucis and the interosseal muscles are nevertheless visible (Figure 4). The coloured arteries and nerves in the medial land lateral sulci of the sole can be clearly seen.

The following comments may be added to the dissection. As compared to the palm, the interosseus muscles of the foot are arranged relative to an axis through the second digit, they are surprisingly small, and their strength 
cannot considerably contribute to the arches. The large muscles in the median eminence, which cover these small muscles, excel in size and probably also in strength. The position and the size of the oblique head of the adductor hallucis combining with the lateral part of the short flexor hallucis suggest their prominent role in holding the transversal and the longitudinal arches in the forefoot. The tendons of the long flexor of the digits stretch in a common layer with the quadratus plantae which fastens the tendons to the calcaneus. About this latter muscle it is assumed that its function is to pull strait the oblique running tendons and to prevent the tips of toes from being bent medial during contraction of their long flexor. The relatively weak quadratus muscle could hardly counteract the strong long flexor of the digits; their pads are, anyhow, pressed against the ground at the beginning of stride. It may rather be assumed that the tendons of the flexor and the quadratus muscles, lying in the cord of the long arch, can be important factors in supporting the arch. On the lateral side, the abductor of the fifth digit belongs to this layer and its position suggests that this muscle also contributes to holding the longitudinal arch. In the next layer the strong short flexor of the digit covers the deep layers. This muscle is virtually extended between the calcaneus and the intermediate phalanges of the digits, and in this position the muscle can act as a tie-beam in the longitudinal arch. Tendons of leg muscles which are attached in the depth of the sole, substantially contribute to the support of the arches. As shown in the present anatomical dissection, that the tendons of the anterior and posterior tibialis muscles attaching on the navicular and on the proximal end of the first metatarsus bones are able to hold very effectively the load on the arches. They are accompanied by the tendon of the long peroneal muscle which runs from lateral to medial across the pedal and is attached to the proxi- mal end of the first metatarsus. Together with the tibial muscles they form a strong and elastic loop underneath the sole. At the highest point of the arches these muscles support the midfoot and supplement the missing medial pilar.

\section{Discussion}

The weight of the body is transferred to the ground through the arches of the foot. The arches function as shock absorbers and provide space for nerves and vessels in the sole. Electromyographic evidence indicates that the intrinsic pedal muscles are strongly active during movement (Wolf et $\mathrm{al}^{10}$ ), but due to technical difficulties there are no data about the role of individual muscles in the support of the arches. Their actions, or at least their potentialities, can be deduced from the geometry of fascicular architecture and attachments, as it has been done in the above consideration. But in structural deductions one must be aware of persisting uncertainty and the conclusion must be taken accordingly. In the followings I want to discuss the role played by muscles and other structural agents in securing the threefold function of the foot: weight bearing, elasticity and motility.

When fitted together, the shape of bones itself presents the structure of a longitudinal and a transversal arch. These arches differ from the conventional structure used in architect. The keystone of the vault, which is a wedgeshaped stone in the centre and stabilizes the arch, is narrower upward and broader downward in the foot. The first cuneiform bone with its broad plantar surface is a reversed keystone in the transverse arch. The supporting pilar is missing from the medial side, the first cuneiform and the navicular bones are floating without any support. Two third of the weight of the body is carried by the dorsal 


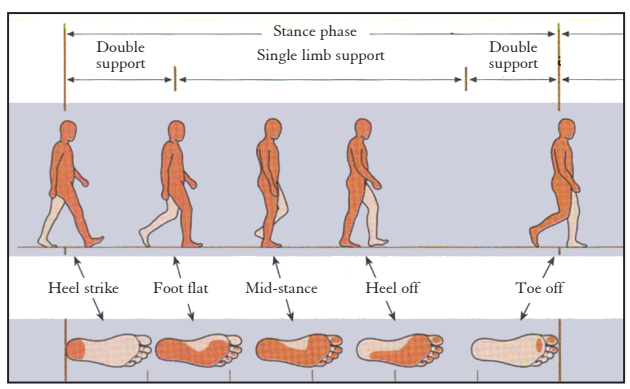

Figure 5. Footprints in different phases of a step (modified from Williams)

one third of the longitudinal arch. This arrangement is very favourable for elasticity and adaptability, but it is frail for weight bearing. These contradictions are solved by the ligaments, but mainly by the muscles.

According to electromyograph studies of Walker ${ }^{8}$, the peculiar shape of the bones and the tight ligaments are sufficient to keep the arches of the foot. During movement everything changes. In a calm, upright position, a person continually produces a balancing movement in a circle of a radius of $25 \mathrm{~mm}$ (Debrunner ${ }^{4}$ ). This movement is performed by the interplay of the flexor-extensor muscles of the leg; and the tendons of three of them (anterior and posterior tibialis, peroneus longus) form a stirrup-like lope underneath the summit of he arches providing a powerful support for them. Cavanagh et $\mathrm{al}^{3}$, and Alexander and Jayes ${ }^{1}$ studied the footprint in different phase of locomotion (Figure 5). They found that following heel strike, the weight of the body is gradually transferred to the forefoot, the sole flattens, and at midstance phase only the medial cavity of the sole is off ground. At the beginning of the heel-off phase, the whole of the body weight shifts onto the heads of the metatarsal bones. The pressure is increased by the muscular thrust when the heel lifts clear of the ground. The sole of the shoe wears off the sooner in this region indicating the heavy load on the fore region of the foot. There are only a few and weak muscles in this region, the interosseus muscles, and it would be their task to hold the arch in the foremost region of the foot. As mentioned above, they receive substantial help from the adductor and short flexor muscles of the hallux and of the fifth digit. The tendons of the long flexor of the digits run in the next layer. It has been also discussed above that the pulling force of the oblique running tendons is straightened by the quadratus plantae. The tendons cross the strong oblique head of the adductor hallux muscle in the former layer. (The tendons of the long flexor is folded forward, therefore this crossing cannot be seen in Figure 4). This X-shaped cross is like the St. Andrew's cross, used by cabinet makers to stabilize the legs of a table. This Andrew's cross can powerfully support the transverse arch which is heavily loaded in the forefoot. This structure is covered by the superficial layer of muscles, the strong short flexor of the digits and the two abductors (hallux and digiti quinti) which lying in the long axis of the foot support the long arch. The four layers of the intrinsic pedal muscles of the foot establish four longitudinal tiebeams in the longitudinal arch, and the different directions in fascicular architectures of muscles form a network within the longitudinal arrangement of the muscle layers. The tendons of the long leg muscles intrude, as it were, this structural arrangement of he pedal muscles, and with their action they suspend the arches in a strong and elastic manner. Patients with flat foot complain about pain in the anterior part of the leg.

\section{The care of the foot}

The human foot is distinguished by its arches from the animal's. Morton ${ }^{7}$ in his recently criticized (Grebing et $\mathrm{Co}^{5}$ ) endeavour suggested that the arches developed by the grad- 
ual strengthen of the second metatarsal bone in the foot of monkeys in evolution. Be it as it may, the complexity of the human foot is much more sophisticated than its evolution could simply be deduced from existing other structures. Due to its complexity, any static and dynamic disorders cause serious complain with such a frequency of occurrence that it can be regarded as a disease of public health concern. If one considers that the foot carries the weight of the body during the larger part of the day, it is no wonder that the day ends with rather uncomfortable feeling not only in the foot but also in the knee and the hip. Modern life style with lack of exercise and walking in shoes on even ground greatly contribute to the development of various problems. An athletic foot, and the foot of nature peoples can stand all these wear and tear, and this refers to the importance of muscles $\left(\right.$ Cavanagh $\left.^{2}\right)$. Fashionable shoes with high heels may cause damages to the foot. In a shoe with extreme high heels the person stands on a slope, the toes slide into the pointed tip of the shoe and body's weight rests on the first and fifth metatarsophalangeal joints. Prolonged wear of such shoes may bring about serious deformities of the toes and shrinkage of the Achiles tendon which causes problems when walking in regular shoes.

These considerations call attention to the importance of foot-care. There are many time-honoured methods and comfortable exercises to keep up a strong and healthy foot. It is very useful to stand, walk and even to dance on tiptoe, jumping and walking on the outer rim of the foot. It is a common exercise to walk barefoot on uneven ground in free nature like nature peoples do. The many stimuli (some of them probably painful) evoke reflective contractions and strengthen the pedal muscles. The performance of little tasks (tying a knot, picking up objects) with the toes increases the skill and strength of the small muscles. A message from the findings of the present works can be a reference to the muscles in the forefoot region. As described above, this part of the foot is double loaded at the preparation to the stance phase and the muscles are very often too weak to support the arches. When they flatten at each step, the load rests on the head of the metatarsal bones, and their repeating compression may cause the painful Morton's neuroma. A regular exercise with forced movements of the toes themselves may strengthen the interosseal muscles and the muscles in the medial and lateral plantar eminences. It is well worth making a little effort for a strong and healthy foot.

\section{Acknowledgements}

I am thankful to Dr. Zoltán Csernátony, Associate Professor and Director of the Department of Orthopaedics, and Dr. Mihály Petkó, Associate Professor in the Department of Anatomy, Medical and Health Science Centre, University of Debrecen. Dr. Mihály Kálmán, Associate Professor in the Department of Anatomy, Semmelweis University, Budapest, provided valuable help with the plastination technique.

\section{REFERENCES}

1. Alexander RMcN, Jayes AS. Fourier analysis of forces exerted in running and walking. J Biochem 1980;13:383-90.
2. Cavanagh PR, Morag E, Boulton AJ, Young MJ, Deggenr KT, Pammaer SE. The relationship of static foot structure to dynamic foot function. J Biochem 1997;30:243-50. 
3. Cavanagh $P R$, Rodgers $M M$, Liboshi A. Pressure distribution under symptom-free feet during barefoot standing. Foot Ankle 1987;7:262-76.

4. Debrunner HV. Funktionelle Anatomie des Fusses. In: E Stoff (ed) Der diabetische Fuss. Berlin, Heidelberg: Springer; 2005. p. 13-24.

5. Grebing BR, Coughlin MJ. Evaluation of Morton's theory of second metatarseal hypertrophy. Brit J Biomed Sci 2004;1375-86.

6. Hagens von $G$. Methods for preserving large sections of biological tissue with polymers. US Patent 4,320,157. filed 1980, issued 1982 .
7. Morton DJ. Evolution of the longitudinal arch of the human foot. J Bone Joint Surg 1924;6: 56-90.

8. Walker IT. The biomechanics of the human foot. Ph.D. Thesis. 1991.

9. Williams PL (ed) Gray's Anatomy. Churchil livingstone, London, 1995.

10. Wolf $P$, Stacoff $A, \operatorname{Lin} A$ et al. Functional units of the human foot. Gait Posture 2008;28: $434-41$.

\section{Tamás Kobezda}

Department of Anatomy, Medical and Health Science Centre, University of Debrecen H-4032 Debrecen, Nagyerdei krt. 98.

(+36) 52 416-392

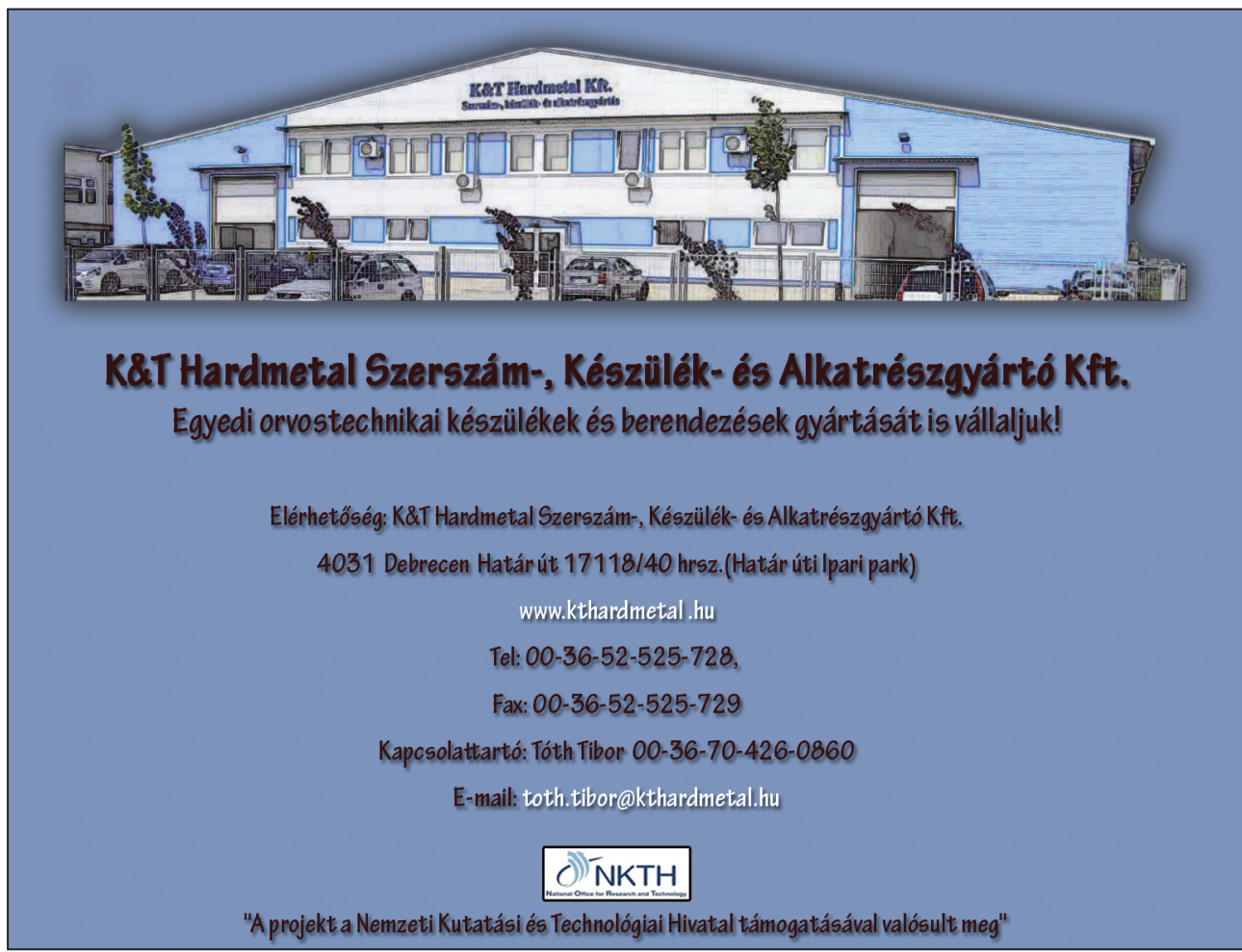

